\title{
Implementación del ABP, PBL y método SCRUM en cursos académicos para desarrollar sistemas informáticos enfocados en fortalecer la región
}

\author{
Ferley Medina-Rojas, John Maicol Nuñez-Santa, Irlesa Indira Sánchez-Medina \& Jaime Malqui Cabrera-Medina \\ Facultad de Ingeniería, Universidad Cooperativa de Colombia, Neiva, Colombia.ferley.medina@campusucc.edu.co, john.nunezs@campusucc.edu.co, \\ irlesa.sanchez@campusucc.edu.co,jaime.cabrera@campusucc.edu.co
}

\begin{abstract}
Resumen - La integración vertical y horizontal de cursos en el currículo académico transmite a los estudiantes conceptos integrales que se fortalecen mediante el aprendizaje basado en problemas y proyectos. Como resultado de implementar estos métodos de aprendizaje se evidencia con un sistema para administrar las labores de producción en cultivos de café utilizando el marco de trabajo SCRUM, el cual establece crear incrementos funcionales con las historias de usuario a través de iteraciones, menos documentación conservando la eficiencia del proyecto, la flexibilidad al cambio de las características, servicios y restricciones de operación.
\end{abstract}

Palabras Clave- Aprendizaje basado en problemas; aprendizaje basado en proyectos, integración vertical y horizontal de cursos, método SCRUM.

Recibido: 2 de diciembre de 2016. Revisado: 15 de mayo de 2017. Aceptado: 6 de junio de 2017

\section{Implementation of ABP, PBL and SCRUM method in academic courses to develop computer systems focused on strengthening the region}

\begin{abstract}
The vertical and horizontal integration of courses in the academic curriculum transmits to students comprehensive concepts that are strengthened through problem-based learning and projects. As a result of implementing these learning methods evidenced a system to manage production work in coffee crops using the Scrum framework work, which states create functional increments through iterations, less documentation preserving the efficiency of the project, flexibility to change the features, services and operating restrictions.
\end{abstract}

Keywords - Problem-based learning, project-based learning, vertical and horizontal integration courses, SCRUM method.

\section{Introducción}

La integración horizontal y vertical de cursos en el currículo académico permite implementar el aprendizaje basado en problemas (ABP) y proyectos (PBL) [1,2]; donde la temática de cada curso complementa los conceptos para solucionar una problemática previamente identificada.
La universidad pedagógica y tecnológica de Colombia hace referencia al ABP en el octavo semestre del programa ingeniería electromecánica, en la asignatura seminario de investigación, en esta el estudiante desarrolla un anteproyecto aplicando los conocimientos adquiridos en cursos anteriores [3]. De igual forma, la facultad de Informática de la Universidad del País Vasco, refieren los resultados del PBL en el curso de Ingeniería de software I, en el cual los estudiantes utilizan el conocimiento integral de los cursos de Programación básica, Programación modular y orientada a objetos, Estructuras de datos y Algoritmos. Al finalizar el periodo académico, el 68\% de estudiantes refieren que su aprendizaje mejoró y se reflejaron calificaciones superiores respecto a los grupos anteriores [4].

En la actualidad, el programa Ingeniería de sistemas de la UCC sede Neiva - Huila ha integrado el ABP durante el curso análisis de sistemas; en las asignaturas diseño de sistemas, base de datos, diseño de investigación e ingeniería de software I y II el PBL [2], el ejercicio está estructurado en identificar y solucionar una problemática posiblemente enfocada a las apuestas productivas del departamento del Huila, estas se contemplan como agroindustria a base de tecnología, turismo, piscícola, minera y energética [5]. Como resultado de esta integración, se muestra un sistema para administrar las labores de producción en cultivos de café utilizando el método SCRUM $[6,7]$.

Cabe destacar que estos métodos de aprendizaje ayudan a fortalecer habilidades transversales como liderazgo, solución a problemas, toma de decisiones, creatividad, adaptación a nuevas situaciones, aprendizaje autónomo, emprendimiento, relaciones interpersonales, trabajo en equipo y razonamiento crítico $[8,9]$.

\section{Marco referencial}

\subsection{Aprendizaje basado en problemas (ABP)}

La implementación del método de aprendizaje basada en problemas (ABP) inició en la Universidad canadiense de

Como citar este artículo: Medina-Rojas, F., Nuñez-Santa, J.M., Sánchez-Medina, I.I. and Cabrera-Medina, J.M., Implementación del ABP, PBL y método SCRUM en cursos académicos para desarrollar sistemas informáticos enfocados en fortalecer la región. Educación en Ingeniería, 12(24), pp. 52-57, Julio, 2017. 
McMaster a mediados de los años 60, donde la facultad de medicina plantea que los profesionales adquieran un conjunto de competencias y habilidades básicas para afrontar dificultades reales, con los conocimientos adquiridos integrados de la investigación [10,11]. El marco de trabajo está estructurado de una serie de procesos encadenados, asociado cada uno con los roles de tutor y estudiante, este último organizado en pequeños grupos [12]. El tutor es el docente del curso encargado de guiar el proceso de aprendizaje transmitiendo la ética profesional y científica [13]. Carmen y Elvira citando a (Moust, Bouhuijs y Schmidt) [10], refieren la siguiente serie de pasos que deben seguir los estudiantes después de identificar el problema y estructurar los equipos de trabajo:

1. Aclarar términos y conceptos desconocidos del enunciado del problema.

2. Definición del problema.

3. Analizar el problema: lluvia de ideas para identificar las posibles causas [14].

4. Analizar las hipótesis planteadas y corroborar si tienen alguna relación con la problemática, posteriormente se organizan destacando las relaciones existentes entre las mismas.

5. Formular objetivos de aprendizaje: Los estudiantes definen qué deben investigar para comprender aún más el problema.

6. Efectuar una investigación exhaustiva, tomando como referencia los vacíos intelectuales identificados en el punto anterior.

7. Realizar un informe final en el cual plasman los conocimientos adquiridos y la conclusión del problema. Igualmente, cada equipo de trabajo socializa dicha información con los demás grupos.

\subsection{Aprendizaje basado en proyectos $(P B L)$}

El aprendizaje basado en proyectos aplicado en las aulas de clase, se enfoca en abordar una problemática presente por grupo de estudiantes y solucionarla. Este método permite al profesional en formación adquirir diferentes competencias y habilidades tales como: el liderazgo, trabajo en equipo, investigación y comunicación. Fernández y Duarte refieren los siguientes procesos a seguir para implementar el ABP en cursos de ingeniería [3]:

- Sensibilización: El tutor describe detalladamente la metodología a los estudiantes, actividades a desarrollar y los roles presentes en cada grupo de trabajo. Al culminar, cada aprendiz expone un problema identificado y se conforman los equipos. Los integrantes del grupo establecen el problema a solucionar y definen los roles que cada uno ejercerá.

- Seguimiento a la Solución del Problema: Es el proceso para realizar seguimiento a la solución planteada durante el semestre y se estructura en cuatro etapas:

- Anteproyecto: El grupo elabora un documento formal donde plasma la identificación del problema, marco referencial y metodología prevista junto con los recursos necesarios para resolverlo.

- Informe de avances: Es un escrito referenciando el progreso de desarrollo del proyecto, debe ser acompañado de una socialización para compartir información acerca de la investigación con los demás equipos de trabajo.

- Informe técnico de la solución: Cada grupo socializa la arquitectura y diseño, justificando, con lenguaje técnico, las herramientas a utilizar. Igualmente, dicha información debe estar recopilada en el documento del proyecto.

- Informe final: Se realiza al finalizar el semestre y socializa el resultado del proyecto y su respectiva documentación.

\subsection{Procesos, modelo, método y metodologías de desarrollo de software}

En ingeniería de software se utilizan los procesos [15] o ciclo de vida [16] de desarrollo que se definen como la comunicación, planeación, modelado, construcción y despliegue de un sistema. La comunicación es la interacción con el cliente para definir e identificar los servicios, características y restricciones de operación del sistema. En la planeación se proyecta el sistema a desarrollar y se identifican los recursos o elementos necesarios; además se instaura un cronograma de actividades. Identificadas las necesidades en la comunicación se realiza el modelado para crear los diagramas UML, definir los componentes o módulos del sistema, establecer el diseño de interfaz y modelo de base de datos (entidades y atributos) [16]. Posteriormente, se efectúa la construcción o codificación del sistema con base a los elementos establecidos en la etapa anterior; durante este proceso, el desarrollador de software realiza pruebas de defectos y depuración de código, al culminar cada componente, se realizan pruebas de integración, operatividad y confiabilidad para comprobar que el sistema en desarrollo cumpla con las características establecidas en las etapas de comunicación y modelado. Finalmente, en la etapa de despliegue, se realiza la evaluación, búsqueda de fallas o errores en el sistema que no se encontraron en las pruebas; la implementación, puesta en marcha del sistema; el mantenimiento son las actualizaciones que se realizan.

Los procesos de desarrollo se estructuran en modelos [15] o paradigmas [16], estos plantean el orden de ejecución, pero no definen cómo se deben realizar, qué herramientas utilizar y quién interviene en cada uno de ellos [17]. Por lo anterior, surgen los métodos que definen el marco de trabajo tomando como referencia la estructura de un modelo y una metodología, en las que se encuentran las tradicionales [18], estas son identificadas por la exigente planeación, documentación, seguimiento y control exhaustivo a cada uno de los procesos que se efectúan de manera independiente, el cliente debe tener claro todas las necesidades en la etapa de comunicación por la carencia de adaptabilidad a cambios. La metodología ágil es opuesta a la anterior, plantea obtener un conjunto de necesidades del cliente más no la totalidad, realizar los procesos de planeación, modelado y construcción en interacciones, a través de bloques de tiempos cortos e integrando al cliente; finalizada cada iteración se entregan los avances para que se efectúe el despliegue. La integración del cliente le permite visionar el sistema en construcción e identificar nuevas características y servicios para orientar el producto a nuevas oportunidades. 


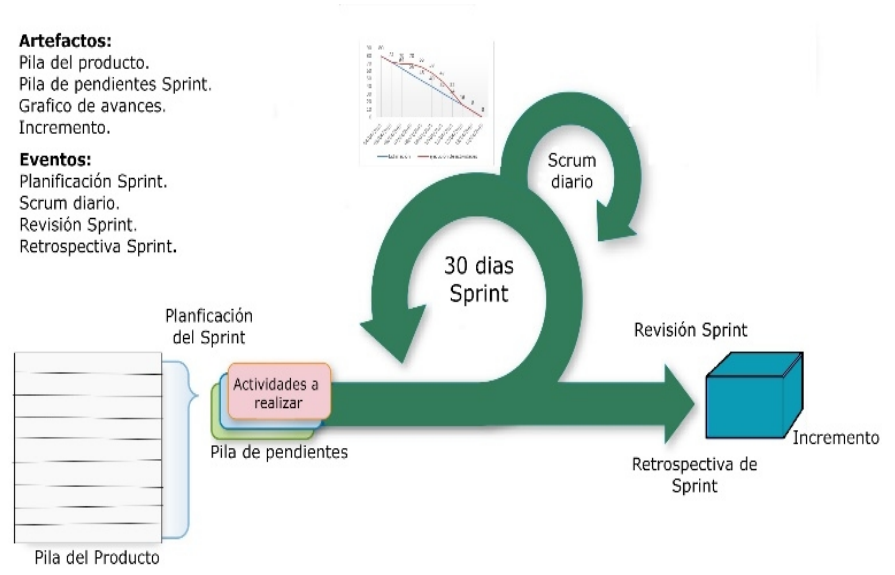

Figura 1. Marco de trabajo SCRUM

Fuente: [20]. Juan palacio “SCRUM Mannager”.

Cesar y Rubén [19] refieren que las metodologías tradicionales son similares al juego de parqués, en el cual las fichas lentamente se llevan al final; cuando una ficha regresa al punto de entrada tiene que realizar nuevamente todo el recorrido. Mientras, las metodologías ágiles las asemejan al ajedrez, donde constantemente hay que adaptarse a los cambios y durante el juego se logran pequeños objetivos.

\subsection{Método de desarrollo SCRUM}

SCRUM es un marco de trabajo basado en la metodología ágil que plantea realizar incrementos funcionales a través de iteraciones llamadas sprint, cada una en un lapso de tiempo menor a 30 días [6]. Además, integra al cliente en los procesos de desarrollo y se adapta con facilidad al cambio de características, servicios y restricciones de operación del sistema [19], el método puntualiza una serie de artefactos, eventos y roles que pueden observarse en la Fig. 1.

La pila del producto contiene el conjunto de características, servicios y restricciones de operación del sistema (historias de usuario) asociadas a una prioridad de ejecución, estimación de tiempo de ejecución y un identificador único [21]. Las historias de usuario que se van a efectuar en un sprint son fragmentadas en tareas, se le asigna el esfuerzo estimado en horas y el responsable de la ejecución, la información se formaliza en la pila de pendientes [22]. En el transcurso de un Sprint se realizan las actividades planeadas, la consumación de cada una se referencia en el gráfico de avances (Burndown Chart), el cual permite visualizar el progreso de ejecución, como se muestra en la Fig. 2.

El eje X representa el número de días del sprint y el eje Y la sumatoria de horas estimadas para desarrollar el conjunto de tareas. Finalizado cada sprint, se plasman en un documento las nuevas características, servicios o restricciones de operación al sistema que se efectuaron.

Los artefactos se formalizan en eventos como la reunión de planificación del sprint, la dinámica consiste en definir qué se va hacer, cómo se hace y cuál es el resultado; se concreta la fecha (día, mes y año) de apertura y cierre del sprint, el objetivo del Sprint e historias de usuario que se realizan para crear el

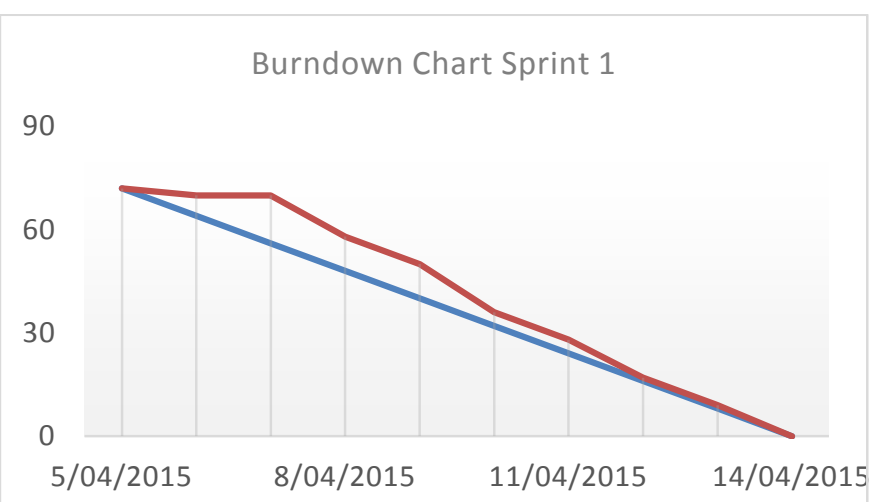

Figura 2. Ejemplo gráfico de avances en un Sprint Fuente: Los autores.

incremento funcional. Después de definir los elementos que conforman la pila de pendientes del sprint, se puntualiza la fecha, hora y minutos lugar de la reunión del SCRUM diario y revisión del sprint. Durante un sprint se realiza la reunión de SCRUM diario antes de cada jornada laboral, allí cada integrante de equipo de trabajo define las tareas a realizar antes de la próxima reunión, se actualiza el grafico de avances y la pila de pendientes. Al culminar un Sprint se efectúa la revisión, allí es informado si el objetivo del Sprint se cumplió, el incremento funcional realizado y actividades pendientes si quedó alguna; además, se realizan ajustes a la pila de producto si el cliente requiere agregar nuevas historias de usuario o modificar las existentes; al finalizar la reunión, el incremento es entregado al cliente para que realice las pruebas de operatividad y se programa la reunión de retrospectiva de sprint, este evento consiste en conocer e identificar las fortalezas y debilidades en las actividades que se desarrollaron en el sprint para implementar planes de mejoras, con la finalidad de transformar las debilidades en fortalezas y optimizar el desarrollo.

Para efectuar los eventos y artefactos, el método establece un equipo de trabajo con los roles de dueño del producto, SCRUM master y equipo de desarrollo [6]. El dueño del producto representa al cliente y usuario final, tiene como responsabilidad gestionar la pila del producto con las historias de usuario, optimizar el valor del producto y trabajo del equipo de desarrollo. Es el responsable que el equipo comprenda y cumpla el marco de trabajo que establece el método con sus respectivas reglas es el maestro SCRUM y el equipo de desarrollo es el conjunto de profesionales que se encarga de realizar las actividades planteadas en un Sprint para crear los incrementos funcionales al sistema.

\section{Metodología}

En el plan de estudio del programa Ingeniería de sistemas (Universidad Cooperativa de Colombia sede Neiva), están integrados de manera horizontal los cursos de Análisis de sistemas, Diseño de sistemas, Base de datos, Diseño de investigación, Ingeniería de software I y II [2]; verticalmente están estructurados Diseño de investigación, Base de datos y Diseño de sistemas. Esta integración tiene el enfoque de utilizar el aprendizaje basada en problemas en la asignatura de Análisis y en las demás, el aprendizaje basado en proyectos orientado en 
solucionar la necesidad identificada, permitiendo a los estudiantes en formación aplicar los conocimientos adquiridos y fortalecer habilidades transversales, liderazgo, solución a problemas, toma de decisiones, creatividad, adaptación a nuevas situaciones, aprendizaje autónomo, emprendimiento, relaciones interpersonales, trabajo en equipo y razonamiento crítico [23].

El curso de análisis de sistemas está estructurado en el tercer semestre académico; donde el docente adquiere el rol de tutor y le solicita a los estudiantes que se agrupen en un máximo de tres personas e identifiquen una problemática de interés a estudiar presente en la región (las apuestas productivas del Huila). Cada integrante aporta información referente al tema para establecer y definir el problema. Identificada la necesidad, se realiza la lluvia de ideas para referir la hipótesis e identificar las variables o datos que se desconocen; estructuran las técnicas de recolección de información con asesoría del tutor para obtener datos concisos. Identificado el problema con las causas, efectos y variables que intervienen, cada equipo de trabajo construye un informe con el resultado final de la investigación y se socializa a los demás compañeros de aula mediante una exposición.

En el cuarto semestre, el estudiante se instruye en el aprendizaje basado en proyectos, los docentes asumen el rol de tutor, realizan la sensibilización de la metodología de aprendizaje, describen la estructura e integración en los cursos de diseño de sistemas, base de datos y diseño de investigación. En el curso diseño de investigación el tutor orienta en los ítems para elaborar el anteproyecto (introducción, objetivos, planteamiento del problema, marco teórico y estado del arte), este ejercicio permite realizar seguimiento al equipo de trabajo, profundizar en el problema, conocer los estudios existentes y respectivas soluciones. En las asignaturas diseño de sistemas y base de datos los estudiantes adquieren conceptos que permiten realizar la arquitectura del sistema o aplicación a desarrollar (modelo UML, diseño de interfaces, integración de módulos y modelo de base de datos). La información se plasma en el anteproyecto con el ítem de arquitectura.

En el quinto semestre, el docente de ingeniería de software I, orienta los conceptos de calidad de software, procesos, modelos, metodología y métodos de desarrollo de software (agiles y tradicionales).

En el siguiente periodo académico, el equipo establece el método de desarrollo de software para la codificación del proyecto (sistema de información, aplicación web o móvil), el tutor induce trabajar con un método ágil, el cual crea incrementos funcionales a través de iteraciones, trabajo en equipo, menos documentación conservando la eficiencia del proyecto, flexibilidad al cambio de las características, servicios y restricciones de operación [6]. Al finalizar el semestre, cada grupo socializa el proyecto, describe la problemática, tecnología utilizada y solución planteada. Además, se espera que el estudiante tenga la capacidad de solucionar y afrontar problemas reales.

\section{Resultados}

En el curso de Análisis de sistemas del tercer semestre, los estudiantes identifican un problema en el sector agrícola, los productores de café registran la información de las labores de producción de manera manual en libros contables y cuando requieren generar reportes de cuentas por pagar (mano de obra), compra de insumos e ingresos obtenidos en ventas de café, cometen equivocaciones al referenciar los datos y demanda tiempo, por la carencia de aplicación amigables para administrar fincas cafeteras. Por lo anterior, se implementa las etapas del ABP para identificar las causas, hipótesis e investigar las variables que intervienen. Al finalizar, concluyen que los productores de café se estructuran en dos grupos, uno con certificaciones y el otros sin ellas, estas certificaciones se determinan como Rainforest (amigable con el medio ambiente), UTZ (buenas prácticas agrícolas y el manejo administrativo de las fincas) y FAIRTRADE (contenido social). Las fincas cafeteras bajo certificaciones realizan el registro de la información de las labores de producción de manera manual y generan reportes, mientras los restantes no efectúan ningún registro, desconociendo la factibilidad de los cultivos e inversiones efectuadas.

Durante el cuarto semestre se estructuro el proyecto denominado "sistema de información para administrar las labores de producción en cultivos de café de la vereda la cumbre (Paicol - Huila)" con el método PBL. Se redactó el anteproyecto, introducción, objetivos, planteamiento del problema, pregunta problema, marco teórico, estado del arte, metodología de investigación e implementaron técnicas de recolección de información para la profundización de la problemática, actividades efectuadas en el curso de Diseño de investigación.

En el curso Diseño de Sistemas los estudiantes aplicaron la teoría adquirida en el curso de análisis, plasmando las actividades que el sistema deberá realizar. Con la orientación del tutor los estudiantes elaboraron el modelo UML (Diagrama de clases, actividades, caso de uso, distribución y componentes). Identificadas las variables que intervienen para realizar la sistematización, se estableció a PostgreSQL como gestor de base de datos, elaborando el modelo entidad relación a través del software Pgmodeler.

En el curso de Ingeniería de Software II se empleó los conocimientos adquiridos en el curso Ingeniería de Software I. Para el desarrollo del proyecto se definió el método SCRUM. La Tabla 1 describe una historia de usuario que se creó con los ítems que establece el marco de trabajo. La Tabla 2 ilustra el artefacto de pila del producto con las historias de usuario organizadas por prioridad de ejecución.

Tabla 1.

Ejemplo de Historia de usuario

\begin{tabular}{|c|c|}
\hline \multicolumn{2}{|r|}{$\begin{array}{lr}\text { Historia de Usuario } & \text { Fecha: } 05-02-2013 \\
\text { Versión: } 2 \\
\end{array}$} \\
\hline No. & $\begin{array}{l}\text { Nombre de historia de Usuario } \\
\end{array}$ \\
\hline 6 & Consultar fincas \\
\hline Usuario & John Maicol Nuñez \\
\hline Descripción & Un usuario puede consultar las fincas asociadas. \\
\hline Condición & Entrada: Nombre de usuario \\
\hline Estimación & 10 horas \\
\hline $\begin{array}{r}\text { Prioridad de } \\
\text { ejecución }\end{array}$ & 10 \\
\hline $\begin{array}{c}\text { Pruebas de } \\
\text { aceptación }\end{array}$ & Mostrar fincas asociadas con la cuenta de usuario \\
\hline
\end{tabular}


Tabla 2.

Ejemplo de pila del producto gestionada con historias de usuario.

\begin{tabular}{clcc}
\hline \multicolumn{2}{c}{ Pila del Producto } & & Fecha: 05-02-2013 \\
Versión: 1 \\
\hline ID & \multicolumn{1}{c}{ Historia de usuario } & Prioridad & $\begin{array}{c}\text { Estimación } \\
\text { horas }\end{array}$ \\
1 & Registro de usuarios & 10 & 25 \\
2 & Actualizar datos de usuario & 10 & 10 \\
3 & Validación de usuarios & 10 & 15 \\
4 & Registro de fincas & 10 & 20 \\
6 & Consultar fincas & 10 & 10 \\
8 & Registro de lotes & 10 & 20 \\
10 & Consultar lotes & 10 & 10 \\
11 & Registro de cultivos & 10 & 20 \\
13 & Consultar Cultivos & 10 & 10 \\
14 & Programar labores y actualizar & 10 & 45 \\
16 & el estado de ejecución & 10 & 25 \\
17 & Rensultar labores & 10 & 25 \\
22 & Constrar empltar empleados & 10 & 20 \\
19 & Asignar mano de obra a una & 10 & 40 \\
& actividad & & \\
\hline
\end{tabular}

Fuente: Los autores.

Tabla 3.

Planificación sprint No. 0

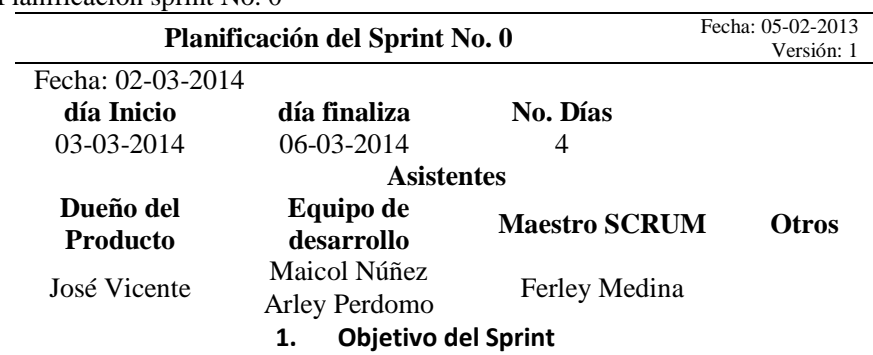

Corroborar que los diagramas de casos de uso, clases, actividades, distribución, y modelo entidad relación cumplan con los parámetros de las historias de usuario.

ID

\section{Historias de usuario \\ Descripción}

Tareas

Observaciones:

Fuente: Los autores.

Tabla 4.

Planificación sprint No. 1

\begin{tabular}{cccc}
\hline \multicolumn{3}{c}{ Planificación del Sprint No. 1 } & Fecha: 05-02-2013 \\
Versión: 1
\end{tabular}

Fuente: Los autores.
En el Sprint 0 se revisó los diagramas UML y modelo entidad relación para corroborar que cumplía con las variables presentes en las historias de usuario. La Tabla 3 muestra la planificación del sprint.

Finalizado el sprint se prosiguió con la planificación del Sprint No. 1, como se muestra en la Tabla 4, el cual contiene las etapas de análisis, diseño, desarrollo y pruebas para las historias de usuario, creando un incremento funcional.

La misma dinámica se efectúa para realización de las demás historias de usuario para la creación de los módulos finca, lotes, cultivos, empleados y labores de producción.

\section{Conclusiones}

El uso del método ABP en la orientación del curso Análisis de Sistema, contribuye a la formación de creatividad en los estudiantes para generar ideas innovadoras orientadas en la resolución de problemas en el ámbito Local, Regional y Nacional.

La integración vertical y horizontal de cursos en la malla curricular del programa ingeniería de Sistemas permite la construcción de ideas que terminan en el desarrollo de proyectos, para lo cual se realizan etapas de seguimiento, elaboración de informes técnicos, entrega de resultado parciales y final. Contribuyendo en la formación de habilidades de liderazgo, solución a problemas, toma de decisiones, adaptación a nuevas situaciones, aprendizaje autónomo y razonamiento crítico a los estudiantes.

El desarrollo de un software implementando un método ágil como es el caso de SCRUM, permite el desarrollo organizado e iterativo de un proyecto, contribuyendo al desarrollo de relaciones interpersonales, responsabilidad, respeto y trabajo en equipo en soluciones productivas.

\section{Referencias}

[1] Delgado, A., Robledo, E. and Márquez, H., Posgrado educación UATX. 2015 [Online]. http://posgradoeducacionuatx.org/pdf2015/B069.pdf

[2] Medina, F. and Rojas, F., El ingeniero de inclusión social, in LACCEI Latin American and Caribbean Conference for Engineering and Technology, Cancun - Mexico, 2013.

[3] Fernández, F.H and Duarte, J., El aprendizaje basado en problemas como estrategia para el desarrollo de competencias específicas en estudiantes de ingeniería, Formación Universitaria, 6(5), pp. 29-38, 2013.

[4] Goñi, A., Ibáñez, J., Iturrioz, J. and Ángel-Vadillo, J., Aprendizaje basado en proyectos usando metodologías ágiles para una asignatura básica de ingeniería del software, in XX JENUI, Oviedo - España, 2014.

[5] Iriarte, C., Rincón, A., Machado-Rojas, L.F. and Chávarro, O., Cámara de Comercio de Neiva. [En línea]. 2015. Disponible en: https://ccneiva.org/servicios-empresariales/empresarios/

[6] Ken, S. and Sutherland, J. (2013, julio) Scrum guides. [En línea]. Disponible en: http://www.scrumguides.org/docs/scrumguide/v1/scrumguide-us.pdf

[7] Medina. F., Sánchez, I., Núñez, J.M. and Cabrera, J., Sistema de información para administrar fincas productoras de café mediante el método de desarrollo SCRUM, in 3er Congreso Internacional AmITIC 2016, Santa Cruz de Bolivia, 2016.

[8] Gundín, O., Fidalgo, R. and García, J.N., El desarrollo de las competencias transversales en magisterio mediante el aprendizaje basado en problemas y el método de caso, Revista de Investigación Educativa, 26(2), pp. 43-44, 2008.

[9] Sanchez Medina, I.I., Medina-Rojas, F and Rojas-Rojas, F., El ingeniero de inclusión social con videojuegos, Educación en Ingeniería, 10(19), pp. 
116-123, 2015.

[10] Vizcarro, C. and Juárez, E., ¿Qué es y cómo funciona el aprendizaje basado en problemas?, in El aprendizaje basado en problemas en la enseñanza universitaria. España: Red de Bibliotecas Universitarias (REBIUN), pp. 17-36. 2008.

[11] Restrepo, B., Aprendizaje basado en problemas (ABP): Una innovación didáctica para la enseñanza universitaria, Educación y Educadores, 8, pp. 9-19, 2005.

[12] Morales, P. and Landa, V., Aprendizaje basado en problemas, Theoria, 13(1), pp. 145-157, 2004.

[13] Meneses, G.A. and Ordosgoitia, C.E., Laboratorio virtual basado en la metodología de aprendizaje basado en problemas, ABP, Educación en Ingenieria, 4(7), pp. 62-73, 2009.

[14] Josep-Solaz, J.J., López, V. and Gómez, A., Aprendizaje basado en problemas en la educación superior: Una metodología necesaria en la formación del profesorado, Didáctica de las Ciencias Experimentales y Sociales, 25, pp. 177-186, 2011.

[15] Roger, S., Pressman, Ingeniería del software un enfoque práctico séptima edición. México D.F: McGraw Hill, 2010, pp. 1-74.

[16] Sommerville, I., Ingeniería de software novena edición. México: Pearson, pp. 1-79.

[17] Cervantes-Ojeda, J. and Gomez-Fuentes, M.del.C., Taxonomía de los modelos y metodologías de desarrollo de software más utilizadas, Universidades, 52, pp. 37-47, 2012.

[18] Trigás, M., (2012, junio) Universitat Oberta de Catalunya. [En línea]. Disponible http://openaccess.uoc.edu/webapps/o2/handle/10609/17885

[19] Rodríguez, C. and Dorado, R., ¿Por qué implementar SCRUM?, ONTARE, 3(1), pp. 125-144, 2015.

[20] Palacio, J. and López, A., Menzinsky, G., Scrum manager. 2016 [Online]. Available at: http://www.scrummanager.net/files/gestion_proyectos_scrum_manager. pdf

[21] Kniberg, H., Scrum and XP from the Trenches: How we do scrum, 2nd edition. United States of America: C4Media, 2015.

[22] Alaimo, D.M., Proyectos ágiles con SCRUM: Flexibilidad, aprendizaje, innovación y colaboración en contextos complejos. Buenos Aires: Ediciones Kleer, 2015.

[23] Sánchez, P. and Blanco, C., Implantación de una metodología de aprendizaje basada en proyectos para una asignatura de Ingeniería del Software, in XVIII JENUI, Ciudad Real, 2012.
F. Medina-Rojas, recibió el título de Ing. de Sistemas en la Universidad Antonio Nariño, Colombia, el título de Esp. en Gestión de Desarrollo Regional en la Universidad Surcolombiana, Colombia, el título de Esp. en Redes de Comunicaciones en la Universidad Cooperativa de Colombia, el título de MSc. en Telemática en la Universidad Central de las Villas, Cuba. Actualmente candidato a $\mathrm{PhD}$ (c) de Ingeniería en la Universidad Pontificia Bolivariana sede Medellín, Colombia. Se desempeña como profesor en el área de ingeniería aplicada de la facultad de ingeniería, Universidad Cooperativa de Colombia, sede Neiva, Colombia.

ORCID: http//orcid.org/0000-0001-8174-6506

J.M Núñez-Santa, recibió el título de Ing. de sistemas en la Universidad Cooperativa de Colombia, sede Neiva, Colombia. ORCID: http//orcid.org/0000-0002-4063-6949.

I.I. Sánchez-Medina, recibió el título de Ing. de Sistemas en 2000, el título de Esp. en Docencia Universitaria en 2005, el título de Esp. en Redes de Comunicaciones en 2008, el título de MSc. en Educación en 2012. Actualmente es estudiante de doctorado en la Universidad Oberta de Catalunya, España, y se desempeña como profesor en el área de ingeniería aplicada de la facultad de ingeniería, Universidad Cooperativa de Colombia, sede Neiva, Colombia. ORCID: http//orcid.org/0000-0002-8840-0708.

J.M. Cabrera-Medina, recibió el título de Lic. en Matemáticas y Física en 1988, el título de Esp. en computación para la docencia en 1995, el título de Esp. en docencia universitaria en 1999, el título de Esp. en tecnologías de la información y la comunicación TIC en educación en 2011, el título de MSc. en tecnologías de la información y la comunicación TIC en educación en 2011. Actualmente es profesor de física de la Facultad de Ingeniería, Universidad Cooperativa de Colombia, sede Neiva, Colombia. ORCID: http//orcid.org/0000-0001-9282-7010. 